\title{
Régimen Jurídico del Comercio Internacional y Derechos Humanos: una Compleja Relación
}

Este artículo se encuentra disponible en www.anuariocdh.uchile.cl

Carlos Fortin*

\section{Introducción}

Uno de los rasgos más distintivos de la globalización económica contemporánea es el esfuerzo -primordialmente por parte de los gobiernos de los países desarrollados- por establecer regímenes jurídico-institucionales para enmarcar el fenómeno, para facilitarlo y para orientarlo en ciertas direcciones. Esas iniciativas han tenido lugar en las diversas áreas de la economía internacional (comercio, inversiones y finanzas) y en todos los planos: multilateral, interregional, regional y bilateral.

A nivel multilateral el más exitoso de esos esfuerzos es en el área del comercio. Los acuerdos resultantes de la Ronda de Uruguay de negociaciones comerciales multilaterales y en particular la creación en 1995 de la Organización Mundial del Comercio (OMC) constituyeron un paso decisivo en la dirección de dar al comercio internacional una estructura de gobernanza dotada de las funciones gubernamentales básicas: legislativa, a través de un mecanismo de adopción de reglas; cuasiejecutiva, en tanto a la OMC le corresponde facilitar la aplicación, administración y funcionamiento de los acuerdos comerciales multilaterales ${ }^{1}$; y cuasijudicial, contenidas en el "Entendimiento sobre Solución de Diferencias" (ESD) que forma parte de los "Acuerdos de la Ronda de Uruguay".

No debe, por consiguiente, causar sorpresa que en los debates sobre la evolución, la problemática actual y las perspectivas del derecho internacional público, haya surgido con fuerza la cuestión de la relación entre el emergente régimen jurídico-institucional del comercio internacional y el otro gran corpus del derecho internacional de la postguerra, el de los derechos humanos. Un conocido estudioso de esta segunda área, el profesor Philip Alston, ha sostenido que "la relación entre los derechos humanos y el comercio es una de las cuestiones centrales que deben enfrentar los juristas internacionales en el comienzo del siglo $\mathrm{XXI}^{\prime 2}$.

* Instituto de Estudios del Desarrollo de la Universidad de Sussex, Reino Unido.

1 Acuerdo por el que se Establece la Organización Mundial del Comercio, artículo III.1. Los textos de todos los documentos jurídicos de la OMC en español se encuentran en el sitio de internet de la organización, en la página: http://www.wto. org/spanish/docs_s/legal_s/legal_s.htm

2 Alston, Philip, "Resisting the Merger and Acquisition of Human Rights by Trade Law: A Reply to Petersmann", The Jean Monnet Programme, Jean Monnet Working Paper 12/02, New York, New York University School of Law, 2002, p. 5, reproducido en European Journal of International Law, Vol. 13, No. 4, 2002, pp. 815-844 (mi traducción; salvo indicación en contrario, todas las traducciones de textos en inglés son mías). Hay ya una abundante literatura sobre el tema. Una completa lista al año 2005 se encuentra en: Monash University, Castan Centre for Human Rights Law, "WTO/Human Rights. Literature Review", September 2005. Disponible en: http://www.law.monash.edu.au/castancentre/projects/wto/ wto-lit-review-05.pdf. Otras contribuciones importantes son: Abbott, Frederick M., Christine Breining-Kaufmann y Thomas Cottier (eds.), International Trade and Human Rights. Foundations and Conceptual Issues, Ann Arbor, Michigan, The University of Michigan Press, 2006; Cottier, Thomas, Joost Pauwelyn y Elisabeth Bürgi Bonanomi (eds.), Human Rights and International Trade, Oxford University Press, 2005. 
El tema es altamente complejo. Incluye tanto aspectos jurídicos como económicos y políticos, y está lleno de dificultades y dilemas en los planos conceptual y empírico. Uno de los más prominentes expertos en el derecho de la Organización Mundial del Comercio, el profesor John H. Jackson, ha cuestionado "si un estudio que relacione los discursos del comercio y los derechos humanos es incluso realizable" ${ }^{3}$, y se ha referido a la complicación adicional resultante de que los lenguajes en la literatura sobre derechos humanos y sobre comercio constituyen una dicotomía de discursos, "como barcos que se cruzan en la noche", la que se ve agravada por el tono de los respectivos discursos, que tiende a ser de propugnación (advocacy).

En este contexto no es, nuevamente, de extrañar que los debates académicos y políticos sobre la cuestión de la relación entre el comercio internacional y los derechos humanos no hayan Ilegado a conclusiones universalmente aceptadas; podría incluso decirse que ni siquiera se ha llegado a acuerdo acerca de los términos en que se plantea el problema, de la definiciones básicas en juego y de los criterios para evaluar las diversas hipótesis propuestas.

En el presente artículo no intentaré dar respuesta final a estas interrogantes. Mi objetivo, mucho más limitado, es hacer un recuento del estado de los debates, procurando introducir alguna claridad acerca de los problemas conceptuales y metodológicos que la temática suscita, y proponer, a partir de las varias contribuciones que serán analizadas, algunas líneas de reflexión, investigación y acción futuras. Mi punto de partida es claro: buscar fórmulas que permitan poner el comercio internacional y las políticas comerciales internacionales al servicio de la realización de los derechos humanos; mi punto de llegada lo es menos por estar compuesto, como se verá, más de preguntas que de respuestas.

\section{Cuestiones conceptuales previas: ¿qué comercio? ¿qué derechos humanos?}

Evidentemente el tema de la relación entre el comercio internacional y los derechos humanos precede con mucho a la creación de la Organización Mundial del Comercio. Sus orígenes se remontan a la Guerra Fría, cuando los gobiernos de los países occidentales decidieron incorporar la cuestión de los derechos humanos en su arsenal de lucha contra el comunismo y su influencia en los países del Tercer Mundo. En esa etapa, la cuestión se planteaba en términos del uso de medidas comerciales para conseguir que otros países respetaran los derechos humanos de sus poblaciones o para sancionar a los violadores. Las medidas eran básicamente la apertura o cierre de mercados de exportación o importación, y los derechos en cuestión eran básicamente los consagrados en el Pacto Internacional de Derechos Civiles y Políticos. Los problemas en ese caso no eran tanto jurídicos como económico-políticos, a saber, ¿cuál es el grado de efectividad de las medidas para conseguir el mejoramiento de los derechos humanos en los países afectados?; ¿cuál es el costo económico y político para el país que impone las medidas?; y, ¿cuál es el costo para la población -inocente- del país afectado? ${ }^{4}$

Esa formulación del problema experimentó un cambio significativo en la década de los noventa. Las causas fueron, por un lado, la entrada en vigencia de los Acuerdos de la Ronda de Uruguay en 1994 y el establecimiento de la OMC, cuyo número de países miembros además creció rápidamente; y por otro, la creciente importancia en el debate internacional de la temática de

\footnotetext{
3 Jackson, John H., "Reflections on the Possible Research Agenda for Exploring the Relationship between Human Rights Norms and International Trade Rules", Capítulo 2 en Abbott, Breining-Kaufmann y Cottier, op.cit., pp. 19, 20.

4 Ver en general Bhagwati, Jagdish, The Wind of the Hundred Days. How Washington Mismanaged Globalization, Cambridge, Mass., The MIT Press, 2002, Capítulo 14, "Trade Linkage and Human Rights", pp. 157-168; y Vazquez, Carlos Manuel, "Trade Sanctions and Human Rights - Past, Present, and Future", Journal of International Economic Law, Vol. 6, No. 4, pp. 797-839.
} 
los derechos económicos, sociales y culturales, culminando con el reconocimiento en 1986 del derecho al desarrollo por la Asamblea General de las Naciones Unidas, ${ }^{5}$, ratificado en 1993 por la Conferencia Mundial de las Naciones Unidas sobre Derechos Humanos de Viena ${ }^{6}$. La OMC, contrariamente a su predecesor, el GATT (Acuerdo General sobre Aranceles Aduaneros y Comercio. GATT por sus siglas en inglés), cuyo mandato se concentraba en la reducción de los aranceles y las barreras al comercio en las fronteras, adquirió un mandato amplio, que incluye áreas como la reglamentación de los servicios, la propiedad intelectual y la inversión, que antes eran de resorte exclusivo de las autoridades nacionales. Era, pues, crecientemente evidente, que la OMC podía tener una influencia importante, incluso decisiva, en las posibilidades de disfrute de los derechos económicos, sociales y culturales y del derecho al desarrollo. La cuestión del comercio y los derechos humanos pasó así a ser definida principalmente en términos de la relación entre el régimen jurídico de la OMC y el régimen de protección internacional de los derechos económicos, sociales y culturales, incluido el derecho al desarrollo ${ }^{7}$.

\section{Comercio, liberalización comercial y derechos humanos}

Esta reformulación agregó importantes complejidades al problema en la medida en que puso en el centro del debate la cuestión más general del impacto del comercio internacional en las perspectivas de desarrollo de los países en desarrollo. El tema es vasto y no es éste el lugar para tratarlo en detalle. Para nuestros fines basta constatar que el mandato de la OMC -en la realidad, y a despecho de algunas lecturas de su carta constitutiva ${ }^{8}$ - es promover la expansión del comercio internacional por medio de la liberalización de las corrientes comerciales y la eliminación de obstáculos o distorsiones interiores al comercio exterior. La teoría económica neoliberal justifica este mandato sosteniendo que la liberalización del comercio internacional es el mecanismo óptimo para maximizar el uso de los recursos, aumentar le eficiencia económica, acelerar el crecimiento y reducir la pobreza. En esta visión, la liberalización del comercio internacional es un instrumento decisivo para la realización de los derechos humanos, en particular los económicos, sociales y culturales, y el desarrollo 9 .

Este enfoque ha sido llevado más lejos en el trabajo de un asiduo comentarista del derecho del GATT y la OMC, el profesor Ernst-Ulrich Petersmann, quien sostiene que la liberalización del comercio y por consiguiente el régimen de la $\mathrm{OMC}$, son en sí mismos una forma de realización de los derechos humanos. Además del argumento utilitario del enfoque neoliberal, Petersmann afirma que hay un valor esencial en reconocer y proteger el derecho a la propiedad y la libertad de contrato $^{10}$.

5 Naciones Unidas, Asamblea General, Declaración sobre el derecho al desarrollo, Res. 41/128 de 4 de diciembre de 1986.

6 Naciones Unidas, Conferencia Mundial de Derechos Humanos, Viena, 14 a 25 de junio de 1993, Declaración y programa de acción de Viena, Doc. A/CONF.157/23, 12 de julio de 1993.

7 La gradual universalización de la OMC significó además que la posibilidad de aplicar sanciones comerciales -esencialmente denegaciones del estatus de nación más favorecida- en razón de violaciones de derechos civiles y políticos se hizo más difícil, y en la opinión de algunos, imposible. Véase el análisis de Vásquez acerca de la posible invocación del artículo XX del GATT 1994 para justificar medidas como las indicadas. Vásquez, op.cit., nota 4 arriba, pp. 816-839.

8 Por ejemplo, Dommen, Caroline, "Safeguarding the Legitimacy of the Multilateral Trading System: The Role of Human Rights Law", Capítulo 6 en Abbott, Cottier y Breining-Kaufmann (eds.), op.cit. nota 2 arriba, pp. 121-124.

9 Sykes, Alan O., "International Trade and Human Rights. An Economic Perspective", Capítulo 4 en Abbott, BreiningKaufmann y Cottier (eds.), op.cit. nota 2 arriba, pp. 69-91.

10 Petersmann, Ernst-Ulrich, "Time for Integrating Human Rights into the Law of Worldwide Organizations. Lessons from European Integration Law for Global Integration Law", The Jean Monnet Programme, Jean Monnet Working Paper 7/01, New York, New York University School of Law, 2001. Reproducido con el título "Time for a United Nations 'Global Compact' for Integrating Human Rights into the Law of Worldwide Organizations: Lessons from European Integration", European Journal of International Law, Vol. 13, No. 3, 2002, pp. 621-650. 
La propuesta de Petersmann ha sido convincentemente refutada tanto desde la perspectiva de derechos humanos (Alston ${ }^{11}$ ) como del derecho de la OMC (Howse ${ }^{12}$, Picciotto ${ }^{13}$ ). Más seriamente para nuestra problemática, parece crecientemente claro que la ciencia económica contemporánea está abandonando el tono de seguridad de la posición neoliberal acerca de los efectos positivos de la liberalización comercial para el crecimiento y la reducción de la pobreza ${ }^{14}$. La visión, más modesta, que gana terreno sostiene que no está probado que más liberalización externa ("apertura") sea en el interés de todos los países o que el mundo necesite ahora un conjunto de reglas mundiales que promuevan la liberalización del mercado mundial; ambas son preguntas abiertas ${ }^{15}$.

La teoría económica no proporciona, por consiguiente, una guía precisa para evaluar la cuestión del impacto de las políticas comerciales y la liberalización del comercio en los derechos humanos; la resolución de posibles conflictos entre ambos sistemas es más materia de análisis político o del juego de preferencias valóricas, que de una resolución técnica.

\section{Derechos económicos, sociales y culturales: problemas de estatus e implementación}

Como ya se ha indicado, el debate actual sobre la relación entre el régimen jurídico de la OMC y los derechos humanos se centra en los derechos económicos, sociales y culturales consagrados en el Pacto Internacional de Derechos Económicos, Sociales y Culturales (PIDESC) ${ }^{16}$ y en otros instrumentos tales como la Convención sobre los Derechos del Niño ${ }^{17}$, o acuerdos ambientales que puedan incidir en derechos económicos, sociales o culturales ${ }^{18}$, así como en el derecho al desarrollo.

Entre los derechos contenidos en el PIDESC aquellos que han suscitado mayor debate en su relación con el régimen de la OMC son el derecho a la salud (Art. 12 PIDESC y Observación general № 14 (2000) del Comité de Derechos Económicos, Sociales y Culturales ${ }^{19}$ ) y el derecho a la alimentación (Art. 11 PIDESC). El problema planteado ha sido el grado en el cual la aplicación de los acuerdos de la OMC puede afectar negativamente el disfrute de esos derechos.

11 Alston, op.cit., nota 2 arriba.

12 Howse, Robert, "Human Rights in the WTO: Whose Rights, What Humanity? Comment on Petersmann", The Jean Monnet Programme, Jean Monnet Working Paper 12/02, New York, New York University School of Law, 2002, reproducido en European Journal of International Law, Vol. 13, No. 3, 2002, pp. 651-660.

13 Picciotto, Sol, "The WTO as a Node of Global Governance: Economic Regulation and Human Rights Discourses", Paper for the Conference on Human Rights and Global Justice, University of Warwick, 29-31 March 2006.

14 Ver Rodrik, Dani, "Trade Policy and Economic Growth: A Skeptic's Guide to the Cross-National Evidence," (with Francisco Rodríguez) en Ben Bernanke and Kenneth S. Rogoff (eds.), Macroeconomics Annual 2000, Cambridge, MA, MIT Press for NBER, 2001; y The Global Governance of Trade as if Development Really Mattered, New York, United Nations Development Programme, 2002.

15 Helleiner, Gerald K. 'Markets, Politics, and the Global Economy: Can the Global Economy Be Civilized? '10 th Raúl Prebisch Lecture, UNCTAD, Geneva, diciembre de 2000, p.5.; Stiglitz, Joseph, Making Globalization Work, The Next Steps to Global Justice, London, Allen Lane, 2006, pp. 72-73.

16 Naciones Unidas, Asamblea General, Resolución 2200 A (XXI), de 16 de diciembre de 1966.

17 Naciones Unidas, Asamblea General, Resolución 44/25, de 20 de noviembre de 1989.

18 Por ejemplo, el Convenio sobre la Biodiversidad Biológica de junio de 1992.

19 Naciones Unidas, Comité de Derechos Económicos, Sociales y Culturales, Cuestiones sustantivas que se plantean en la aplicación del Pacto Internacional de Derechos Económicos, Sociales y Culturales, Observación general Nº 14 (2000), El derecho al disfrute del más alto nivel posible de salud (artículo 12 del Pacto Internacional de Derechos Económicos, Sociales y Culturales), doc. E/C.12/2000/4. (General Comments). Ver también Naciones Unidas, Consejo Económico y Social, Comisión de Derechos Humanos, Subcomisión de Promoción y Protección de los Derechos Humanos, Consecuencias para los derechos humanos del Acuerdo sobre los Aspectos de los Derechos de Propiedad Intelectual Relacionados con el Comercio. Informe de la Alta Comisionada, Doc. E/CN.4/Sub.2/2001/13, 27 de junio de 2001. 
El derecho al desarrollo requiere de una breve explicación adicional, ya que ha sido objeto de críticas en términos de su contenido específico, los derechos y obligaciones que crea, y los titulares de esos derechos y obligaciones ${ }^{20}$.

La Declaración sobre el Derecho al Desarrollo identifica como titulares del derecho a la persona humana, los pueblos y los Estados. El contenido específico del derecho en tanto su titular es la persona humana, es la participación en el desarrollo, en el triple sentido de tomar parte en el proceso, de contribuir a él y de gozar de sus frutos. La participación en el proceso, a su vez, implica el estar informado acerca de él (la transparencia), poder exigir cuentas a los responsables del proceso (la responsabilidad o accountability) y en última instancia influir en las decisiones a través de las cuales se realiza. En este sentido el derecho al desarrollo es lo que se ha denominado un derecho de participación, lo que lo hace diferente de los derechos económicos, sociales y culturales precedentes, donde el objetivo es que el individuo tenga acceso a ciertos bienes sociales -educación, salud, trabajo- sin que importe su participación en el proceso por el cual dichos bienes sociales se hacen accesibles ${ }^{21}$.

Estrechamente ligado a este elemento está un segundo componente del contenido del derecho al desarrollo en tanto derecho del individuo. Él es el derecho a que los beneficios y los costos del desarrollo se repartan equitativamente, no sólo en su estadio final sino a lo largo del proceso.

Estos dos elementos - participación y equidad en el proceso de desarrollo- se dan también con respecto al segundo titular del derecho al desarrollo identificado en la declaración, a saber los pueblos. Sus correlatos en este caso son el derecho a la libre determinación y el derecho a la plena soberanía sobre sus riquezas y recursos naturales.

Con respecto al tercer titular del derecho, los Estados, que de acuerdo con el artículo 2 de la Declaración tienen el derecho "a formular políticas de desarrollo nacional adecuadas, con el fin de mejorar constantemente el bienestar de la población entera y de todos los individuos", la visión prevaleciente es que en dicho caso el Estado es titular del derecho en tanto agente de la población entera y de todos los individuos, y respecto de otros agentes - no solamente otros Estados sino otros agentes económicos internacionales, tales como las empresas transnacionales- que pudieran tratar de negar o constreñir la capacidad del Estado de formular dichas políticas ${ }^{22}$.

El énfasis en los derechos económicos, sociales y culturales y el derecho al desarrollo plantea una problemática especial en la relación entre los derechos humanos y el régimen del comercio internacional. Estos derechos son de los llamados derechos positivos, que requieren de acción por parte del Estado para realizarlos. Esa acción a su vez exige recursos, y en un contexto de recursos escasos plantea la difícil cuestión de la jerarquía de los derechos humanos y de su relatividad. ¿Cómo decidir cuál de los derechos, y a qué nivel, tratar de satisfacer en desmedro de otros? ¿Cómo enfrentar el argumento de que determinadas políticas comerciales que pueden aparecer como temporalmente perjudiciales para ciertos derechos humanos se justifican por sus efectos positivos en esos derechos en el mediano o largo plazo?

20 Fortin, Carlos, "El derecho al desarrollo: ensayo de conceptualización", Revista Jurídica de la Universidad de Puerto Rico, Vol. 76, No. 1, 2008 (en prensa).

21 Fortin, op.cit. Un buen compendio del trabajo reciente sobre el derecho al desarrollo es el volumen producto del Simposio Nobel sobre el Derecho al Desarrollo y los Derechos Humanos en el Desarrollo que tuvo lugar en Oslo en octubre de 2003: Andreassen, Bard A. y Stephen P. Marks (eds.) Development as a Human Right. Legal, Political and Economic Dimensions, Cambridge, Mass., Harvard School of Public Health, François-Xavier Bagnoud Center for Health and Human Rights, 2006.

22 Orford, Anne, "Globalization and the Right to Development", en Alston, P. (ed.), People's Rights, Oxford University Press, 2001, p.137; Alston, P., "The Shortcomings of a 'Garfield the Cat' Approach to the Right to Development", California Western International Law Journal, vol. 15, 1985. 
Una respuesta a estas preguntas que se encuentra cada vez más frecuentemente en la literatura es la que sostiene que las normas de derechos humanos son perentorias (son normas de jus cogens) o crean obligaciones erga omnes, y por consiguiente tienen prioridad con respecto a los tratados internacionales ${ }^{23}$. De ser correcto, este enfoque resolvería cualquier posible conflicto entre los derechos humanos y el régimen de comercio internacional; no así, por cierto, la cuestión de la prioridad en la realización de los diversos derechos humanos: en la mayor parte de los casos, la propuesta es la de considerar como parte del jus cogens todos los derechos consagrados en la Declaración Universal de los Derechos Humanos, que incluyen los derechos económicos, sociales y culturales. La propuesta se ha extendido también a considerar el derecho al desarrollo como parte del jus cogens ${ }^{24}$.

La tesis de que todos los derechos contenidos en la Declaración Universal de los Derechos Humanos han pasado a formar parte del jus cogens es muy difícil de defender. Reflejando el pensamiento de la mayoría de los estudiosos del tema, Alston afirma que "hay, en realidad, relativamente pocos derechos que han logrado el estatus de jus cogens", y los que menciona son las prohibiciones contra el genocidio y la esclavitud. La Comisión de Derecho Internacional en su comentario al artículo 26, Cumplimiento de las normas perentorias, de los "Artículos sobre la Responsabilidad de los Estados por Actos Internacionalmente Ilícitos", afirma que los criterios para identificar las normas perentorias del derecho internacional en general son estrictos y menciona como normas perentorias que son claramente aceptadas y reconocidas las prohibiciones de la agresión, el genocidio, la esclavitud, la discriminación racial, los crímenes contra la humanidad y la tortura, y el derecho a la autodeterminación ${ }^{25}$.

A su vez, la Corte Internacional de Justicia en el caso Barcelona Traction menciona como obligaciones erga omnes a las prohibiciones de agresión, de genocidio y las que resultan de principios y reglas sobre los derechos básicos de la persona humana, entre ellas la protección contra la esclavitud y la discriminación racial ${ }^{26}$.

En estas circunstancias no es realmente posible plantear que todos los derechos económicos, sociales y culturales sean normas de jus cogens. Por la inversa, ha sido el propio Comité de Derechos Económicos, Sociales y Culturales el que ha reconocido, a través del concepto de "progresiva efectividad", que estos derechos sólo pueden implementarse gradualmente y teniendo debida cuenta de la disponibilidad de recursos. Por otro lado, esto no significa que los derechos puedan simplemente relegarse a un futuro indeterminado; el Comité enfatiza que corresponde a cada Estado Parte una obligación mínima de asegurar la satisfacción de por lo menos niveles esenciales de cada uno de los derechos ${ }^{27}$.

\section{La normatividad de la Organización Mundial del Comercio y los derechos humanos}

De lo expuesto se sigue que la cuestión de las prioridades en la implementación de los derechos económicos, sociales y culturales se mantiene como un tema abierto, susceptible de diversas

\footnotetext{
23 Para una formulación representativa de esta posición, ver Allmand, Warren, "Trading in Human Rights - The Primacy of Human Rights in International Law", Quebec, Rights \& Democracy (International Centre for Human Rights and Democratic Development), March 24, 1999, disponible en internet: http://www.dd-rd.ca/site/publications/index.php?i $\mathrm{d}=1297$ \&subsection=catalogue\#r16

24 Bedjaoui, Mohammed, "Some Unorthodox Reflections on the 'Right to Development'", en Snyder, Francis y Peter Slinn (eds.), International Law of Development, Abingdon, Professional Books, 1987, pp. 87-116.

25 Yearbook of the International Law Commission, 2001, vol. II, Part Two.

26 Barcelona Traction, Light and Power Company, Limited, Second Phase. Judgment, I.C.J. Reports, 1970, párrafos 33-34.

27 Comité de Derechos Económicos, Sociales y Culturales. Observación general № 3 La índole de las obligaciones de los Estados Partes (párrafo 1 del artículo 2 del Pacto) párrafos 9 y 10.
} 
interpretaciones sobre la base de consideraciones políticas, ideológicas y valóricas. Otro tanto sucede con la cuestión de la prioridad substantiva, en caso de conflicto, entre la protección de esos derechos y la implementación de ciertas políticas comerciales.

Este último tema, sin embargo, tiene un ángulo más específicamente jurídico, susceptible, por consiguiente, de un tratamiento más riguroso. Ello porque, como ya he indicado, la OMC representó un avance importante con respecto al GATT en la dirección de proporcionar una estructura jurídica al comercio internacional, incluida su justiciabilidad. En ese proceso es fundamental la adopción, como parte de los acuerdos de la Ronda de Uruguay, del Entendimiento sobre Solución de Diferencias ${ }^{28}$, y un breve resumen de sus rasgos esenciales es necesario aquí.

\section{El mecanismo de solución de diferencias de la OMC}

EI ESD establece un mecanismo para conocer de las reclamaciones que cualquier miembro de la OMC pueda plantear de conformidad a los artículos XXII y XXIII del "Acuerdo General sobre Aranceles y Comercio" (GATT 1994), en particular, cuando considere que una ventaja resultante para ella del Acuerdo se halle anulada o menoscabada, o que el cumplimiento de uno de los objetivos del Acuerdo se halle comprometido, a consecuencia del incumplimiento de sus obligaciones por otro Estado miembro, o de la aplicación por otro Estado miembro de una medida, aunque no sea contraria al Acuerdo, o de otra situación ${ }^{29}$. El ESD amplió las bases de las posibles reclamaciones al establecer que ellas pueden referirse a cualquiera de los acuerdos de la Ronda de Uruguay ("los acuerdos abarcados"), a saber el Acuerdo por el que se establece la Organización Mundial del Comercio, doce "Acuerdos Multilaterales sobre el Comercio de Mercancías", el "Acuerdo General sobre el Comercio de Servicios", el "Acuerdo sobre los Aspectos de los Derechos de Propiedad Intelectual relacionados con el Comercio" y el propio "Entendimiento sobre Solución de Diferencias" 30 .

El Órgano de Solución de Diferencias (OSD) tiene jurisdicción obligatoria y exclusiva sobre las diferencias entre miembros de la OMC derivadas de la aplicación de los acuerdos abarcados ${ }^{31}$. El Entendimiento establece que el mecanismo tiene por objeto preservar los derechos y obligaciones de los miembros en el marco de los acuerdos abarcados y aclarar las disposiciones vigentes de dichos acuerdos de conformidad con las normas usuales de interpretación del derecho internacional público, y agrega que las recomendaciones y resoluciones del OSD no pueden entrañar el aumento o la reducción de los derechos y obligaciones establecidos en los acuerdos abarcados ${ }^{32}$.

Se plantea por consiguiente la cuestión de la relación entre la normativa de la OMC y la normativa internacional de derechos humanos. ¿Es el derecho de los derechos humanos aplicable a las diferencias entre miembros de la OMC en materias relativas a los acuerdos de la OMC? ¿Puede un miembro de la OMC eximirse del cumplimiento de obligaciones establecidas en los acuerdos de la OMC en razón de normas de derechos humanos? ¿Puede un miembro de la OMC utilizar medidas comerciales para alcanzar objetivos de derechos humanos en el territorio de otro miembro?

\footnotetext{
28 Entendimiento relativo a las normas y procedimientos por los que se rige la solución de diferencias, Anexo 2 del Acuerdo por el que establece la Organización Mundial del Comercio.

29 Acuerdo General sobre Aranceles y Comercio (GATT de 1994), Art. XXIII, Anulación o menoscabo, párrafo 1, incisos a) b) y c).

30 ESD, Apéndice 1.

31 ESD, Artículo 23.

32 ESD, Artículo 3, párrafo 2; y Artículo 19, párrafo 2.
} 
La abundante literatura sobre el tema de la relación entre el régimen de la OMC y otros regímenes internacionales, incluido el de derechos humanos ${ }^{33}$, coincide en estructurar el análisis en términos de tres cuestiones: (1) el derecho aplicable a las relaciones comerciales entre miembros de la OMC; (2) la jurisdicción y competencia del Órgano de Solución de Diferencias de la OMC; y (3) el uso de normas externas a la OMC en la interpretación de los acuerdos abarcados. La clasificación, aunque en teoría clara y neta, resulta más difusa en el análisis concreto, y los analistas además difieren en elementos esenciales del problema.

Con relación a la cuestión del derecho aplicable, las posiciones van desde los que sostienen, en un extremo, que los acuerdos abarcados constituyen lex specialis en el sentido del Artículo 55 de los "Artículos sobre Responsabilidad de los Estados por Actos Internacionalmente Ilícitos" de la Comisión de Derecho Internacional y son por consiguiente el único derecho aplicable (con excepción de normas de jus cogens); la OMC en esta visión sería un "régimen autocontenido"34; a los que, como Pauwelyn en el otro extremo, sostienen que el régimen de la OMC es parte integral del derecho internacional público, y por consiguiente este último se aplica de acuerdo con las reglas de conflicto pertinentes aceptadas por las partes, las que pueden estar contenidas en los propios acuerdos de la OMC, en el tratado que se invoca para justificar el no cumplimiento de la obligación de la $\mathrm{OMC}$, o en el derecho internacional general. Si la regla de conflicto indica que la norma externa a la OMC prevalece sobre la norma de OMC, esta última no debe ser aplicada. Ello porque, al aceptar la regla conflicto aplicable, las partes han aceptado también la posibilidad de que la norma de la OMC sea desplazada por la norma externa.

De lo dicho ya se puede apreciar la dificultad de separar la temática del derecho aplicable de aquella de la jurisdicción del Órgano de Solución de Diferencias, con su jurisdicción obligatoria y exclusiva. Todos los analistas están de acuerdo que el OSD no puede conocer sino de reclamaciones basadas en los acuerdos abarcados; que no puede ejecutar sino las normas contenidas en dichos acuerdos; y que no puede alterar los derechos y obligaciones de las partes emanados de los acuerdos abarcados. Ello obviamente refuerza la posición de los que sostienen que la OMC es un régimen autocontenido; aun si un Estado miembro tuviera derecho a invocar disposiciones externas a la OMC para regir sus relaciones comerciales con otros Estados miembros, ello sólo podría hacerse efectivo recurriendo a otras instancias de adjudicación internacionales.

El análisis se complica aún más al incorporar el tercer elemento antes indicado, a saber la cuestión de la interpretación por el OSD de los acuerdos abarcados. Como ya se dijo, de acuerdo al artículo 3.2 del ESD es función del OSD aclarar las disposiciones vigentes de los acuerdos abarcados "de conformidad con las normas usuales de interpretación del derecho internacional

33 Las siguientes son algunas de las contribuciones más importantes: Bartels, Lorand, 'Applicable Law in WTO Dispute Settlement Proceedings', Journal of World Trade, Vol. 35, No. 3, 2001, pp. 499-519; Marceau, Gabrielle, "WTO Dispute Settlement and Human Rights", European Journal of International Law, Volume 13, Number 4, September 2002, pp. 753-814, reproducido como Capítulo 10 en Abbott, Breining-Kaufmann y Cottier (eds.), op.cit., nota 2 arriba, pp. 181-260; Marceau, Gabrielle, 'Conflicts of Norms and Conflicts of Jurisdictions: The Relationship between the WTO Agreement and MEAs and other Treaties", Journal of World Trade Vol. 35, No. 6, 2001, pp 1081-1131; Palmeter, David and Petro C. Mavroidis, 'The WTO Legal System: Sources of Law', American Journal of International Law, Vol. 92, 1998, pp. 398-413; Palmeter, David y Petros C. Mavroidis, Dispute Settlement in the World Trade Organization.Practice and Procedure, Cambridge University Press, 2004; Pauwelyn, Joost, 'The Role of Public International Law in the WTO: How Far Can We Go?', American Journal of International Law Vol. 95, 2001, pp. 535-578; Pauwelyn, Joost, Conflict of Norms in Public International Law: How WTO Law Relates to Other Bodies of International Law, Cambridge University Press, 2003; Trachtman, Joel, 'The Domain of WTO Dispute Resolution', Harvard International Law Journal, Vol. 40, 1999, pp. 333-377; Weiss, Friedl, "The limits of the WTO: facing non-trade issues", Capítulo 11 en Sacerdoti, Georgio, Alan Yanovich y Jan Bohanes, The WTO at Ten. The Contribution of the Dispute Settlement System, Cambridge University Press, 2006, pp. 155-190.

34 Simma, Bruno, 'Self-Contained Regimes', 16 Netherlands Yearbook of International Law (1985). Esta es la posición de Trachtman, aceptada también con algunas reservas por Marceau. 
público". Esta disposición ha sido interpretada por el Órgano de Apelación con referencia a los artículos 31 y 32 de la Convención de Viena sobre el Derecho de los Tratados ${ }^{35}$. Como se recordará, el inciso c) del párrafo 3 del artículo 31 establece que en la interpretación, junto con el texto, deberá tenerse en cuenta "toda norma pertinente de derecho internacional aplicable en las relaciones entre las partes".

Por esta vía es posible, por consiguiente, el reintroducir consideraciones relativas a normativas externas a la OMC para interpretar la normativa de la OMC, siempre que la normativa externa sea aplicable en las relaciones entre las partes, es decir, que esté contenida en tratados de que ellas sean parte, o forme parte del derecho consuetudinario internacional general o de los principios generales del derecho. Esa posibilidad se da no solamente por el hecho sabido de que todo texto jurídico requiere de alguna interpretación, sino porque el sistema de la OMC contiene un sinnúmero de disposiciones que establecen excepciones al cumplimiento de las obligaciones contenidas en los acuerdos. Las excepciones generales están contenidas en el artículo XX del GATT 1994 y aunque ninguna se refiere explícitamente a los derechos humanos, varias tocan áreas conexas, tales como la protección de la salud y la vida o de los recursos naturales. Algunas de estas excepciones aparecen también en el acuerdo general sobre el comercio de servicios, y en forma más restringida en el acuerdo sobre propiedad intelectual.

Más directamente pertinentes a la cuestión de los derechos económicos, sociales y culturales y el derecho al desarrollo son las excepciones por razones económicas contenidas en el GATT 1994. El artículo XVIII, titulado Ayuda del Estado para favorecer el desarrollo económico, autoriza a los Estados miembros menos desarrollados a adoptar medidas de comercio exterior en contravención a sus obligaciones en la OMC para apoyar o proteger a la producción nacional o para enfrentar problemas de balanza de pagos resultantes de la ejecución de sus programas de desarrollo económico. El artículo establece un procedimiento detallado y complejo para ejercitar este derecho, que en la práctica rara vez ha sido invocado en el GATT o la OMC.

El artículo XII del GATT 1994 contiene una excepción general, no restringida a los países menos desarrollados, por razones de balanza de pagos. Nuevamente, el procedimiento es complejo, y en particular exige que el país suprima las medidas "tan pronto como deje de estar justificado su establecimiento o mantenimiento". En el único caso que ha llegado al OSD, los Estados Unidos cuestionaron la continuación de restricciones aplicadas por India por estimar que ya no se justificaban $^{36}$. El Órgano de Apelación dio la razón a Estados Unidos contra las objeciones del gobierno de la India, que sostenía que la supresión de las medidas exigía que la India modificara su política de desarrollo, lo que no estaba dispuesta a hacer. Para su decisión el Órgano de Apelación se basó en un informe del Fondo Monetario Internacional que afirmó que la India necesitaba modificar solamente su política macroeconómica, no su política de desarrollo, para hacer posible la eliminación de las restricciones.

El fallo ilustra las dificultades para el uso de las excepciones contenidas en los acuerdos de la OMC, en la medida en que el OSD tiende a favorecer las opciones liberalizadoras con respecto a las que impliquen restricciones. El profesor Robert Howse de la Escuela de Derecho de la Universidad de Michigan ha planteado además que este caso ilustra bien lo que sería el efecto de incorporar la referencia al derecho al desarrollo en la decisión del OSD. De haber sido así, sostiene, el Órgano

\footnotetext{
35 Organización Mundial del Comercio, Estados Unidos - Pautas para la gasolina reformulada y convencional, Informe del Órgano de Apelación, WT/DS2/AB/R, 29 de abril de 1996; y Japón - Impuestos sobre las bebidas alcohólicas, Informe del Órgano de Apelación, WT/DS8/AB/R, WT/DS10/AB/R, WT/DS11/AB/R, 4 de octubre de 1996.

36 Organización Mundial del Comercio, India - Restricciones cuantitativas a la importación de productos agrícolas, textiles e industriales. Informe del Órgano de Apelación, $W T / D S 90 / A B / R, 23$ de agosto de 1999.
} 
de Apelación: (1) no habría aceptado que la tecnocracia de un organismo internacional dijera la última palabra sobre lo que es la "política de desarrollo"; (2) no habría establecido una distinción tajante entre políticas macroeconómicas y políticas de desarrollo; aquéllas evidentemente tienen efectos sobre los recursos que el país necesita para el desarrollo; (3) habría solicitado y tomado en cuenta los puntos de vista de una gama amplia de instituciones y actores sociales, al menos los de las organizaciones internacionales con un mandato de desarrollo, como el Programa de las Naciones Unidas para el Desarrollo (PNUD) y la Conferencia de las Naciones Unidas sobre Comercio y Desarrollo (UNCTAD); y 4) en última instancia habría aceptado que el artículo XVIII reconoce el derecho de la India y de los indios de decidir su propio camino en materia de política de desarrollo, y no es una invitación al OSD para reexaminar a partir de cero el juicio de la India acerca de que remover las restricciones exige una modificación de la política de desarrollo ${ }^{37}$.

Por otro lado, es de notar que un caso relacionado con el medio ambiente ${ }^{38}$ el Órgano de Apelación apareció dispuesto tanto a descansar en tratados externos a los de la OMC como a aceptar que las percepciones y valoraciones cambiantes de la comunidad deben ser tomadas en cuenta a la hora de decidir casos en la OMC. Ello podría abrir la posibilidad de que consideraciones basadas en convenciones de derechos humanos y en la evolución de la conciencia de la comunidad internacional en materia de derechos humanos pudiera asimismo tener un impacto.

\section{Los derechos humanos en la OMC: el derecho a la salud y los derechos de propiedad intelectual}

Este último fue precisamente el caso en otro conflicto reciente en que se enfrentaron argumentos de legalidad de la OMC con argumentos de derechos humanos. El asunto, relacionado con los medicamentos para tratar el VIH/SIDA, tiene que ver con dos disposiciones del Acuerdo de la OMC sobre Aspectos de los Derechos de Propiedad Intelectual Relacionados con el Comercio (ADPIC), y su incidencia en la OMC no fue a través del OSD -el único proceso judicial que se inició en relación con él fue en los tribunales de la República de Sudáfrica- sino a través de un proceso político-diplomático que culminó en una enmienda del Acuerdo de los ADPIC.

Las disposiciones son el artículo 31 sobre licencia obligatoria y el artículo 6 sobre agotamiento del derecho de propiedad intelectual ${ }^{39}$. El artículo 31 permite el uso de una patente sin autorización del titular cuando "el potencial usuario haya intentado obtener la autorización del titular de los derechos en términos y condiciones comerciales razonables y esos intentos no hayan surtido efecto en un plazo prudencial" ${ }^{40}$. El artículo agrega que el Estado puede eximir de este requisito "en caso de emergencia nacional o en otras circunstancias de extrema urgencia, o en los casos de uso público no comercial". El inciso f) del artículo establece que "se autorizarán esos usos principalmente para abastecer el mercado interno del miembro que autorice tales usos" y el inciso

37 Howse, Robert, "Mainstreaming the Right to Development in WTO", South Bulletin, No. 85, agosto 2004.

38 Organización Mundial del Comercio, Estados Unidos - Prohibición de las importaciones de determinados camarones y productos del camarón, Informe del Órgano de Apelación, WT/DS58/AB/R 12 de octubre de 1998.

39 Sobre el Acuerdo de los ADPIC en general, ver UNCTAD/ICTSD, Resource Book on TRIPS and Development: An authoritative and practical guide to the TRIPS Agreement, Cambridge, Cambridge University Press, 2005. Internet: http:// www.iprsonline.org/unctadictsd/ResourceBookIndex.htm; y Comisión sobre Derechos de Propiedad Intelectual, Integrando los derechos de propiedad intelectual y la política del desarrollo, Londres, Department for International Development, 2002, internet: http://www.iprcommission.org/papers/pdfs/Multi_Lingual_Documents/Multi_Lingual_Main_Report/ DFID_Main_Report_Spanish_RR.pdf. Sobre ADPIC y derechos humanos, ver Abbott, Frederick M., "TRIPS and Human Rights: Preliminary Reflections", Capítulo 8 en Abbott, Breining-Kaufmann y Cottier, op.cit., nota 2 arriba, pp. 145-169; y Naciones Unidas, Consejo Económico y Social, Comisión de Derechos Humanos, Consecuencias para los derechos humanos, op.cit. nota 19 arriba.

40 Organización Mundial del Comercio, Acuerdo sobre los Aspectos de la Propiedad Intelectual Relacionados con el Comercio, Artículo 31 Otros usos sin autorización del titular de los derechos. 
h) agrega que "el titular de los derechos recibirá una remuneración adecuada según las circunstancias propias de cada caso, habida cuenta del valor económico de la autorización".

Por su parte el artículo 6 se refiere al agotamiento del derecho, una característica propia de la propiedad intelectual consistente en que el derecho del titular de la patente a recibir un pago por el uso de la misma se agota con la primera venta del producto respectivo. El problema aquí es si el derecho se agota en todo el mundo con la primera venta en el país en que se produce la mercadería bajo patente (agotamiento internacional) o si es necesario pagar nuevamente al titular en cada primera venta en otros países que reconozcan la patente (agotamiento nacional). El artículo 6 establece que "para los efectos de la solución de diferencias en el marco del presente Acuerdo, a reserva de lo dispuesto en los artículos 3 y 4 no se hará uso de ninguna disposición del presente Acuerdo en relación con la cuestión del agotamiento de los derechos de propiedad intelectual" 41 (los artículos 3 y 4 contienen las obligaciones de trato nacional y de nación más favorecida, respectivamente). El artículo ha sido interpretado por la gran mayoría de los estudiosos como autorizando a cada Estado el decidir si el agotamiento es nacional o internacional (en este último caso otro país que reconoce la patente podría importar el producto del país productor sin tener que volver a pagar patente, la llamada "importación paralela") ${ }^{42}$. Sin embargo, la cuestión no se ha planteado hasta ahora ante el Órgano de Solución de Diferencias, y existía una opinión minoritaria entre los comentaristas en el sentido de que el artículo 6 no resuelve la cuestión del agotamiento nacional o internacional.

Sujeta a algunas complejidades en las que no podemos entrar aquí, la aplicación conjunta de estos dos artículo podría, en principio, permitir a un país que reconoce una patente (pero no está dispuesto a pagar al titular por encima de lo que el país estima es una suma razonable) el importar el producto en forma genérica de un país que no reconoce la patente o en el cual el producto es producido bajo licencia obligatoria y además ha adoptado el régimen de agotamiento internacional. Dicho uso estaba, sin embargo, sujeto a restricciones que podían hacerlo muy difícil, si no ilusorio: en el caso de la licencia obligatoria, la restricción consistía en que el uso debía ser "principalmente" en el país que otorga la licencia; en el caso de la importación paralela, la restricción residía en la percibida ambigüedad del artículo 6 en relación con el agotamiento internacional.

El problema se planteó específicamente en el caso de la República de Sudáfrica en relación con los medicamentos antirretrovirales para el tratamiento del VIH/SIDA. Hasta el año 2005 ellos eran producidos en la India -que no reconocía la patente- a un precio que en algún momento representó menos del $3 \%$ del precio demandado por las compañías farmacéuticas transnacionales ${ }^{43}$. En esas condiciones, Sudáfrica, que tiene el mayor número de casos de VIH/SIDA del mundo y no tiene la capacidad de producir esos medicamentos bajo licencia obligatoria, aprobó en diciembre de 1997 una modificación a la Ley de Medicamentos y Substancias Relacionadas que autorizaba al Ministro de Salud a decidir que "Ios derechos con respecto a cualquier medicamento bajo patente otorgada en la República no se extenderán a actos con respecto a dicho medicamento que haya sido puesto en el mercado por el dueño del medicamento o con su consentimiento" (Art. 15[c] $)^{44}$.

Aunque la formulación del texto es un tanto ambigua, las compañías farmacéuticas de los Estados Unidos lo interpretaron como una autorización para otorgar licencias obligatorias y efectuar

$41 \mathrm{Ibid}$, Artículo 6 Agotamiento de los derechos.

42 Ver v UNCTAD/ICTSD, op.cit., nota 39 arriba, Capítulo 5: Exhaustion of Rights, pp. 92ss.

43 Oxfam. TRIPS and Public Health. The next battle. Oxfam Briefing Paper 15, marzo de 2002, p. 8. Internet: http://www. oxfam.org/en/files/pp020325_trips_health.pdf/download

44 La descripción de los eventos en Sudáfrica y los Estados Unidos que sigue está basada en Chung, Theresa June, Shocking the Conscience of the World: International Norms and the Access to AIDS Treatment in South Africa, J.D. Thesis, Harvard Law School, 2002. 
importaciones paralelas, y lanzaron de inmediato una intensa campaña de presión ante el gobierno y el Congreso de los Estado Unidos para conseguir su derogación. En una primera etapa las compañías consiguieron que las autoridades norteamericanas aceptaran su planteamiento; un informe del Departamento de Estado de febrero de 1999 consigna que "[t]odos los organismos correspondientes del gobierno de los Estados Unidos [...] han estado empeñados en una campaña asidua y concertada para persuadir al gobierno de Sudáfrica de que retire o modifique las disposiciones del artículo 15(c) que pensamos son incompatibles con las obligaciones y compromisos de Sudáfrica bajo el acuerdo de la OMC sobre Aspectos de los Derechos de Propiedad Intelectual Relacionados con el Comercio (ADPIC)"45. A la campaña se sumaron también los gobiernos de Alemania, Francia y Suiza. En 1998, 40 compañías farmacéuticas transnacionales y la Asociación de Investigación y Manufactureros Farmacéuticos de América (PhRMA) iniciaron un juicio ante los tribunales sudafricanos alegando la inconstitucionalidad del artículo 15(c).

La campaña de las compañías farmacéuticas trascendió a la opinión pública de los países desarrollados y de los países en desarrollo y provocó una reacción igualmente intensa de parte de las organizaciones no gubernamentales interesadas en salud pública y de la sociedad civil en general. Ello y la proximidad de la elección presidencial en los Estados Unidos, en que el candidato demócrata era el Vicepresidente Gore, determinó un cambio en la posición del gobierno norteamericano, que esencialmente cesó en su campaña ante el gobierno sudafricano. Por esa misma presión las compañías se desistieron del juicio en Sudáfrica en abril de 2001, y el problema se trasladó a la OMC, donde los países en desarrollo demandaron la eliminación de las restricciones al uso de licencias obligatorias e importación paralela, demanda que fue resistida por los países desarrollados, especialmente los Estados Unidos, Suiza y Canadá.

La discusión en la OMC fue larga y compleja. Los argumentos no fueron de naturaleza exegética sino de política económica y social. Los países en desarrollo argumentaron que el derecho humano de acceso a la salud debía tener prioridad con respecto a las expectativas de ganancias de las compañías farmacéuticas; éstas replicaron que el respeto a los derechos de propiedad intelectual garantizaba la continuación del esfuerzo de investigación de nuevos medicamentos así como la calidad de los medicamentos, y por consiguiente aseguraba el derecho a la salud en el largo plazo ${ }^{46}$.

El debate atravesó por varias etapas. En la Declaración Ministerial de la Reunión Ministerial de Doha de noviembre de 2001 se incluyó un párrafo que adopta una línea equidistante entre las dos posiciones ("Ir]ecalcamos la importancia que atribuimos a que el Acuerdo relativo a los Aspectos de los Derechos de Propiedad Intelectual relacionados con el Comercio (Acuerdo sobre los ADPIC) se interprete y aplique de manera que apoye la salud pública, promoviendo tanto el acceso a los medicamentos existentes como la investigación y desarrollo de nuevos medicamentos") ${ }^{47}$; además se aprobó una Declaración sobre los ADPIC y Salud Pública ${ }^{48}$ que reiteró el derecho de los países de otorgar licencias obligatorias de conformidad al artículo 31 y confirmó que el

45 United States Department of State, U.S. Government Efforts to Negotiate the Repeal, Termination, or Withdrawal of 15(C) of the South African Medicines and Related Substances Act of 1965. Internet: http://www.cptech.org/ip/health/sa/stdeptfeb51999.html

46 International Federation of Pharmaceutical Manufacturers Associations, "TRIPS, Pharmaceuticals and Developing Countries: Implications for Care Access, Drug Quality and Drug Development", Ginebra, 2000.

47 Organización Mundial del Comercio, Conferencia Ministerial de Doha, Declaración Ministerial, 14 de noviembre de 2001, párrafo 17.

48 Organización Mundial del Comercio, Conferencia Ministerial de Doha, Declaración Ministerial de Doha sobre los ADPIC y la salud pública, 14 de noviembre de 2001. 
artículo 6 autoriza a cada país a decidir su propio régimen de agotamiento de los derechos, pero que dejó abierta para discusión posterior la cuestión de la exportación de productos producidos bajo licencia obligatoria.

Dos años más tarde, en agosto del 2003 una Decisión del Consejo General de la OMC ${ }^{49}$ aprobó una exención que elimina las limitaciones a las exportaciones en el marco de licencias obligatorias para países que no pueden fabricar por sí mismos los productos farmacéuticos. Esta exención, inicialmente temporal, se hizo permanente con la aprobación en diciembre de $2005^{50}$ de una enmienda al Acuerdo de los ADPIC que incorpora un nuevo artículo 31 bis que consagra el contenido de la Decisión de 2003. El texto, en su parte esencial, reza:

Artículo 31 bis

1. Las obligaciones que corresponden a un Miembro exportador en virtud del apartado f) del artículo 31 no serán aplicables con respecto a la concesión por ese Miembro de una licencia obligatoria en la medida necesaria para la producción de un producto o productos farmacéuticos y su exportación a un Miembro o Miembros importadores habilitados de conformidad con los términos que se enuncian en el párrafo 2 del Anexo del presente Acuerdo.

Con esta decisión la OMC aceptó incorporar a su régimen jurídico la visión propuesta por los países en desarrollo según la cual el derecho humano a la salud prevalece sobre las pretensiones de compañías transnacionales, apoyadas por sus gobiernos, basadas en una concepción particular de las características y el impacto económico de la propiedad intelectual y su expresión jurídica, los derechos de los ADPIC. En esa medida, representa un paso importante en el esfuerzo de incorporar una perspectiva de derechos humanos al régimen jurídico del comercio internacional. Al mismo tiempo, el hecho de que sea un caso, en muchos sentidos, único y que aun así haya requerido de un proceso largo y tortuoso para llegar a buen término debe servir de señal de alerta acerca de las muchas y serias dificultades de la empresa.

\section{Conclusión}

La cuestión de la relación entre el régimen jurídico del comercio internacional y los derechos humanos es sin duda central a las preocupaciones tanto de juristas como de especialistas en comercio y desarrollo. En ella, el jurista está confrontado al problema de la unidad y la coherencia del derecho internacional público ${ }^{51}$. ¿Cuán lejos estamos del ideal del derecho internacional como un cuerpo unificado, cuyas diversas ramas se interpenetren, suplementen y refuercen, y con normas de conflicto claras generalmente aceptadas? ¿Son las tendencias contemporáneas en la evolución del derecho internacional en la dirección de la coherencia y la unidad? ¿O hay elementos -particularmente la emergencia, con gran dinamismo, del derecho internacional económico- que sugieren un futuro de fragmentación, de "regímenes autocontenidos" y de carencia de premisas y valores compartidos? ¿Qué podemos -qué debemos- hacer ante la situación?

49 Organización Mundial del Comercio, Consejo General Decisión sobre la aplicación del párrafo 6 de la Declaración de Doha relativa al Acuerdo sobre los ADPIC y la Salud Pública, 30 de agosto de 2003.

50 Organización Mundial del Comercio, Consejo General Decisión sobre la enmienda del Acuerdo sobre los ADPIC, 6 de diciembre de 2005.

51 Ver Marceau, Gabrielle, 'A Call for Coherence in International Law', Journal of World Trade, Vol. 33, No. 1, 1999, pp. 87-152; Trachtman, Joel, "The Missing Link: Coherence and Poverty in the WTO", Journal of International Economic Law, Vol. 8, No. 3, September 2005, pp. 611-622; Hsu, Kristy, "Towards Enhanced International Coherence: Current Work under the WTO and Possible Solutions", 2006 Asia WTO Research Network Conference, Taipei, April 24-25, 2006. Disponible en internet: http://www.rieti.go.jp/wto-c/060726-13.pdf 
Para el estudioso del comercio y desarrollo, las preguntas son igualmente trascendentales. ¿Cuáles son los parámetros básicos de las políticas económicas nacionales e internacionales que pueden generar bienestar, desarrollo e igualdad? ${ }^{52}$ ¿Cuál es lugar del comercio internacional y de las políticas comerciales en esos parámetros? ¿Cómo se pueden compatibilizar la escasez de recursos característica del subdesarrollo con la necesidad de proteger los derechos humanos básicos? ¿Puede un "enfoque del desarrollo basado en derechos" ayudar? ${ }^{53}$.

Para ninguna de estas interrogantes hay respuestas categóricas. En el análisis precedente he tratado de ilustrar algunos de los dilemas que esas interrogantes plantean sobre la base del examen de una temática específica, la relación del régimen de la Organización Mundial del Comercio con los derechos humanos económicos, sociales y culturales y el derecho al desarrollo. He tratado de sugerir que aun en ese caso específico las respuestas sólo pueden ser provisorias. La OMC como régimen jurídico-institucional no está fácilmente abierta a la presencia de la temática de los derechos humanos; sin embargo, el régimen ofrece algunas posibilidades que pueden ser aprovechadas en mayor medida de lo que lo han sido hasta ahora. A su vez, el cuerpo de derecho internacional referido a los derechos económicos, sociales y culturales y al derecho al desarrollo también requiere de una reflexión profunda para abordar de manera concreta los problemas de escasez, de gradualidad y progresividad, de jerarquización y compatibilización, temas que son también cruciales a la reflexión sobre el desarrollo. Las comunidades intelectuales y políticas que se ocupan de estas diversas problemáticas no han tenido hasta ahora un verdadero diálogo. El progreso en esta trascendental materia va a depender en gran medida de que empiecen a tenerlo.

52 Stiglitz, Jospeh E., Globalization and its Discontents, New York, W.W. Norton \& Company, 2002.

53 Ver, Uvin, Peter, Human Rights and Development, Bloomfield, CT, Kamatian Press, 2004, que contiene una exhaustiva bibliografía. 